\title{
The FEM Wiki project: a conversion of a training resource for field epidemiologists into a collaborative Web 2.0 portal
}

\author{
Patty Kostkova, Martin Szomszor \\ City eHealth Research Centre, City University, \\ London, UK, EC1V 0HB \\ \{patty@soi.city.ac.uk, martin.szomszor.1@city.ac.uk\}
}

\begin{abstract}
While an ever increasing popularity of online wiki platforms, usertagging tools, blogs, and forums is the core characteristic of the Web 2.0 era, converting an existing high-quality training module into a collaborative online space for an active community of practice $(\mathrm{CoP})$ while preserving its quality approval processes is a challenging task. This is the aim of the ECDC-funded Field Epidemiology Manual (FEM) wiki project, based on training resources organized in 17 chapters developed for the European EPIET epidemiology training programme. This paper describes the challenges, solutions, and development processes behind the FEM wiki portal - an online collaborative Web 2.0 platform taking advantage of the user-generated input while preserving the structure, editorial processes and style of the existing FEM manual. We describe the need for ECDC-recognised content and discuss the editorial roles developed in this European project but applicable to any other training resource converted into an online wiki platform.
\end{abstract}

Keywords: Web 2.0, Wiki, Community of Practise, collaborative tools

\section{Introduction}

In order to protect public from emerging infectious disease threats arising from international trade, travel, and bioterrorism, there is a need for rapid internationally coordinated response. This requires instant information dissemination at national and international levels and better coordination of expertise. The Internet and Web 2.0 tools now provide the vehicles to support such rapid communication and the opportunity to create an infrastructure that will foster online communities of practise $(\mathrm{CoP})$, enable online training, mobilisation of knowledge and scientific expertise to support field investigation, encourage knowledge sharing, collaboration and capacity building across Europe.

Web 2.0 significantly contributes to social networking, widening participation and collaboration in healthcare. Social networking is central to the healthcare system in $21^{\text {st }}$ century enabling explicit connections between people, online collaboration, knowledge sharing and collaborative filtering processes [1]. 


\subsection{FEM wiki Project background}

Based on an establish EC funded training programme European Program for Intervention Epidemiology Training (EPIET ${ }^{1}$ ), currently run by ECDC, the aim of the FEM wiki project is to develop an online platform disseminating EPIET training resources to a wider audience using online collaborative methods drawing from the expertise of original authors, EPIET facilitators, EPIET fellows and other experts in the community. Based on the initial 17 chapters constituting the core resources for the EPIET training program, the online FEM wiki portal provides a collaborative space for a community of practice around field epidemiology, enabling discussions, translations into national languages, sharing of case studies, multimedia items and lecture slides.

Rather than aiming only on the EPIET community, the target audience for the FEM wiki will become global, including FETP's in EU, Tephinet ${ }^{2}$, as well as experts working in the area of communicable disease prevention and control (e.g. Microbiology Reference Laboratory network, TESSY users etc).

\subsection{Web 2.0 and social networks}

Communities of practice $(\mathrm{CoP})$ are bringing together a group of people sharing the same "concern or a set of problems" [2] who often have a different level of expertise [3]. The domain creates a common ground and identity for those who care about the domain and subsequently continually maintain and develop the actual practice [4]. In particular, online $\mathrm{CoP}$ in learning environments have been widely investigated [5]. Their success seems to be driven by the popularity of the domain itself and active members or moderators, so called "hubs" who drive the discussions [6]. In the first year of Wikipedia's existence, launched in January 2001, over 20,000 encyclopaedia entries were created at a rate of over 1,500 articles per month. In May 2010, the total number of articles in Wikipedia reached 3.3 million [7].

In addition to $\mathrm{CoP}$, where the connecting feature is the domain itself, social networks have created a new paradigm for online interaction (e.g. Facebook, Twitter, LinkedIn, Flicker, YouTube, etc), supporting users in the creation of online profiles that in turn facilitate communication and networking [8]. Led by Facebook, with more than 400 million active users and 50\% of active users logging on in any given day [9], social networks allow members to advertise their professional background, photographs, expertise and professional interests, as well as join discussions and groups, engage in synchronous communication (IM/chat), post updates about their status and discuss topical issues in various forums. The marriage of CoP, Wikis, and Social Networks to facilitate learning and collaboration are the principal aims of the the FEM wiki project.

\section{Roles in an online editing - who is the author?}

The editorial process and roles of editors and reviewers in a traditional publishing industry are well established. However, the introduction of collaborative usergenerated content, an intrinsic part of this project, created the need for a novel

\footnotetext{
${ }^{1} \mathrm{http}: / /$ www.epiet.org/index.html

${ }^{2} \mathrm{http}: / /$ tephinet.org
} 
approach to acknowledgement and author recognition. Since this project utilises existing content created for the EPIET programme rather than creating online content from scratch, the role definitions and graphical representation, adapted from Wikipedia, are listed below:

- Editor An editor is someone who selects, prepares language, images, tables, and graphs, and organizes the content of the FEM wiki.

- Author An author is anyone who contributed to the initial content of a chapter, or subsequently adds new content during the community involvement phase. A 'Page Contributors' widget, in Figure 1, which lists all the contributors. Editors and Original Authors will receive their own sections.

- Reviewer A reviewer is only required for Chapters where editors are also the initial authors, to ensure a peer-review process, however, any editor can invite colleagues to comment. Reviewers are responsible for providing feedback on the content, advising the editors of errors in the content, of other content that should be included, etc. Any reviewers will be listed in the Page Information widget under contributors.

- Contributor Any users who subsequently edit the page will be added to the Contributors list. This will be ranked according to the substantiality of their comments.

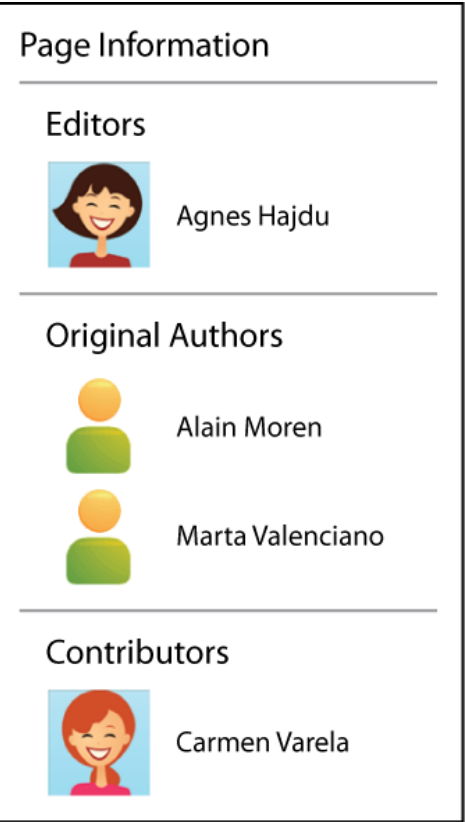

Fig. 1 - Authors, Editors, and Contributors Widget

\section{ECDC-recognised content versus user-generated}

FEM consists of 17 chapters, written by approved/commissioned authors, intended to be read and lectured in a strictly defined order and in a controlled manner. Converting this text into an online wiki portal, preserving the structure of the Manual and control over ECDC-recognised quality while simultaneously supporting all expected collaborative features and an unsolicited creation of user-generated content is a novel task and the main challenge of this project.

In order to ensure the desirable quality of the content and responsibility for updating the existing chapters, an Editorial Board (EB) was created to oversee the editorial process on the EPIET chapters and manage the transition to an online wiki. The EB consists of European experts in the field of epidemiology, typically those involved in the initial EPIET programme as authors, lectures, fellows or facilitators. A discussion about each specific chapter, driving up the quality of the content, is managed in a dedicated forum created for each chapter and will be available to public 
users. Therefore, the core content on the portal is an online version of the FEM wiki chapters so called "ECDC-recognised version", approved by chapter Editors and branded with ECDC logo. To preserve the structure of the manual these will be available from the top FEM table of content together with a link to the dedicated forum.

Once the editorial process is finished and the site is open for public, the creative power of the online community of epidemiologists accessing the public portal will be leveraged by allowing anyone to contribute and update the content. As common in other wiki platforms, the FEM wiki portal will support tracking the changes made to pages, keeping a complete history of all versions of the content. In order to distinguish between the quality-assured ECDC-recognised versions, approved by the $\mathrm{EB}$, future changes by public users superseding the recognised version, are accessible through the page history, but will not affect the ECDC-recognised version. The main Table of Contents page also provides an index of the different version available, as well as links to their corresponding forums, as depicted is Figure 2.

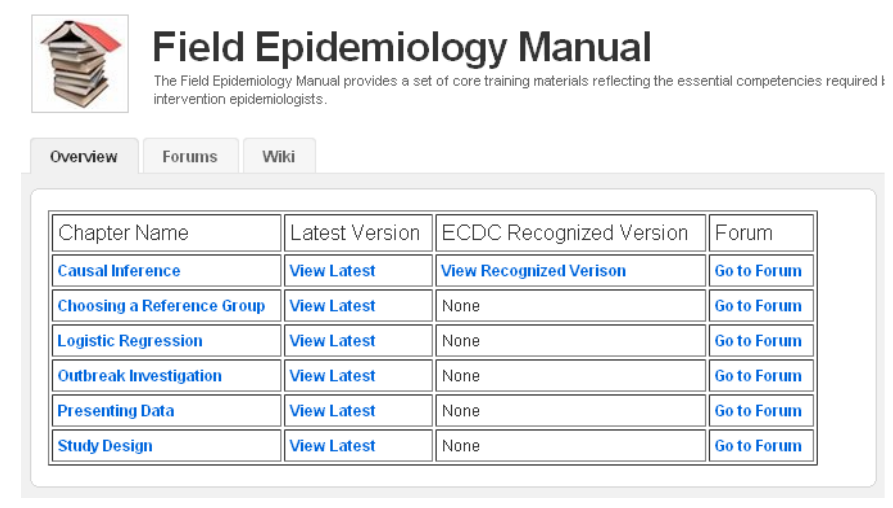

Fig. 2 - Table of Contents Page

From a user's perspective, when viewing any page, a widget will be displayed to inform users of which version of the document they are viewing. If they are viewing the ECDC Recognised version (Figure 3), a link will be given to the most recent version. If they are viewing the most recent version (Figure 4), they will be advised that the content has not been verified, and a link to the ECDC Recognised version will be given.

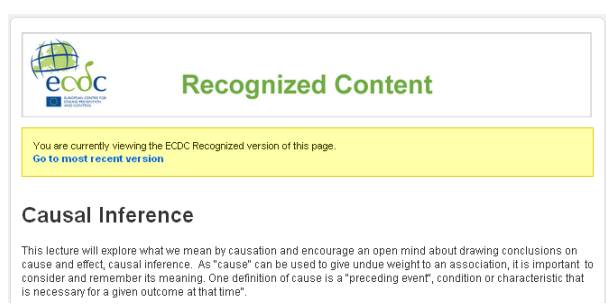

Fig. 3 - ECDC Recognised Content

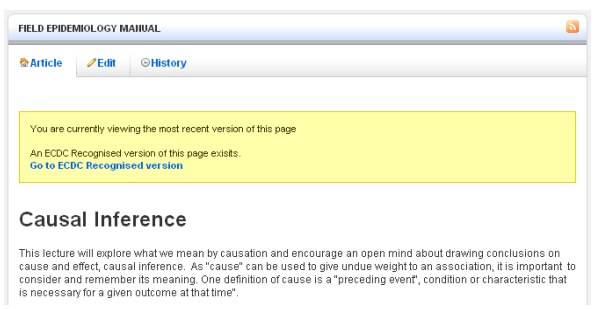

Fig. 4 - Latest Version 


\section{Creating an online wiki from a textbook}

Since collaborative efforts and an organic evolution of content are intrinsically part of wiki tools, the single-file EPIET chapters needed to be reorganised to reflect the nature of an online environment. Unlike the current readers of the paper manual, users might read the whole chapter for training purposes, read only the introduction about a certain concept, or visit only a single page found via a search engine. Finding the right balance between preserving the FEM chapters as consistent training modules, while providing platform for organic growth of the content and random access has been a demanding task.

In order to preserve the nature and style of the FEM wiki, a Home Page for each chapter is manually created and styled to contain a list of learning objectives, an introduction, and a series of content pages (as depicted in Figure 5). Definitions of terms and concepts are cross-linked between pages (using hyperlinks) so readers can choose to find out more information at the click of a button.

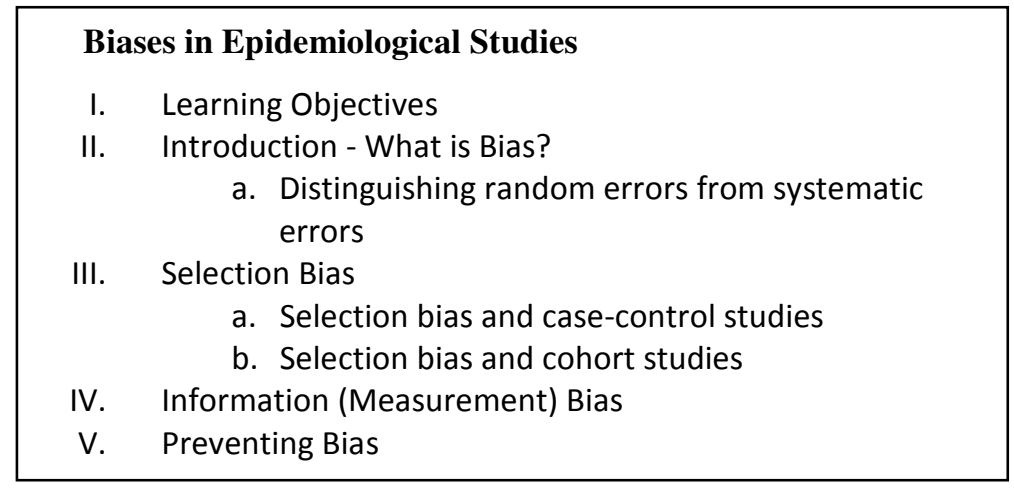

Fig. 5 - Chapter Homepage Template

\section{Implementation}

The FEM wiki portal resides on a single domain: http://www.femwiki.com and is being developed using the Telligent Community (TC) platform ${ }^{3}$. This choice was an outcome of a requirements gathering and feasibility study conducted in 2009 [10] based on the following criteria: licence cost, development platform compatibility with ECDC ICT environment, future use as an e-learning platform and the requirement for a single-sign on. In this section we describe the structure, implementation issues, project status and future work.

\subsection{Platform and Website Structure}

Figure 6 illustrates the structure of the FEM wiki portal depicting additional features such as the Help Pages, Forums etc. The Field Epidemiology Manual (large box) contains the core content of the site, namely the content pages, their corresponding forums. In addition, we also provide an editors space (bottom box) - a private wiki

\footnotetext{
${ }^{3} \mathrm{http}: / /$ telligent.com
} 
and Forum to host materials relevant only to editors (such as best practices, style guidelines, etc) and to hold discussions about the editorial process.

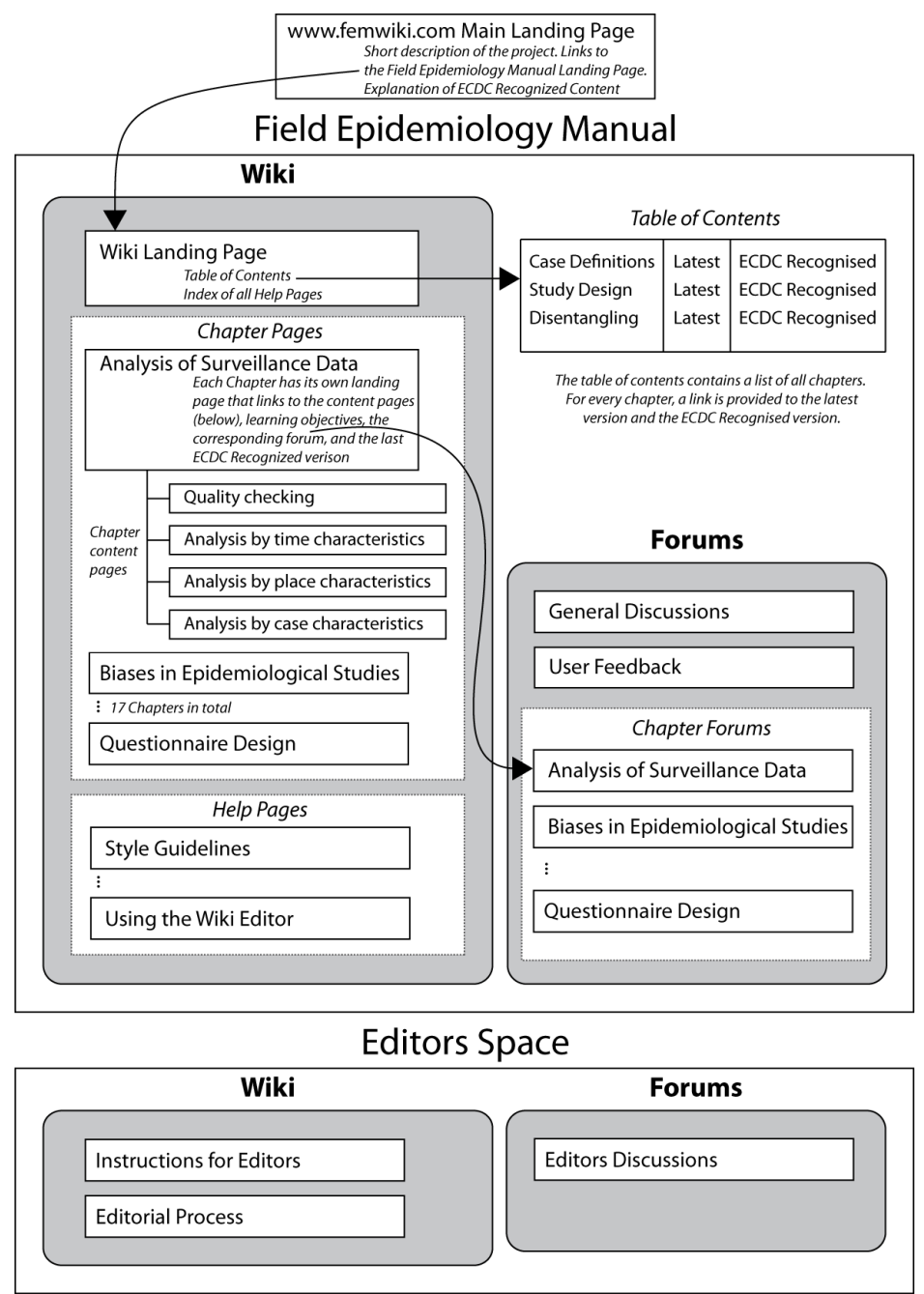

Fig. 6: FEM wiki structure

\subsection{Technical Issues}

The FEM Wiki requires a number of features that are not supported by the TC platform. One of these is the requirement to maintain an index of ECDC-recognised versions. While the TC wiki does maintain a history of page versions (much like other platform such as MediaWiki ${ }^{4}$ do), it does not allow particular version to be given a special status. We have extended the default functionality to support this, maintaining

\footnotetext{
${ }^{4}$ http://www.mediawiki.org
} 
an index of the version last designated ECDC-recognised. Links are provided at the top of every page to allow users to switch between versions easily and understand which version of a page they are currently viewing. Only editors have the privileges to specify when a page is ECDC-recognised.

A second requirement is to associate each page with a set of Editors, Authors, and Contributors (as outline above in Section 2). While it is convention for a wiki to keep track of all the page contributors, they do not usually display them. Given the nature of the FEM material, we believe it important to display prominently the names of those who have created and edited the material in order to establish trust in the scientific content of the page. The Editor(s) and original authors are assigned manually to each page, whereas the list of contributors is built dynamically based on those who have added content to the page.

\subsection{Project Status and Future Plans}

The portal is currently in an "Editing Phase" and is therefore not open to public. This will allow the Editorial Board members to work on converting their content to the online structure. The portal will be officially launched and opened to the public at the ESCAIDE 2010 conference in November 2010. This will mark a "Community Involvement Phase" when users will be able to comment, improve and update the content of chapters. As discussed above, although these might be the latest versions they will not have the status of ECDC-recognised unless the Editor of the chapter approves the changes. This is envisaged to be an annual process or an exercise conducted on a more regular basis should the importance or the number of user changes requires it. When editors are editing and modifying pages, they will have the option to lock (or freeze) the page so that others cannot make changes. Figure 7 depicts the long-term lifecycle.

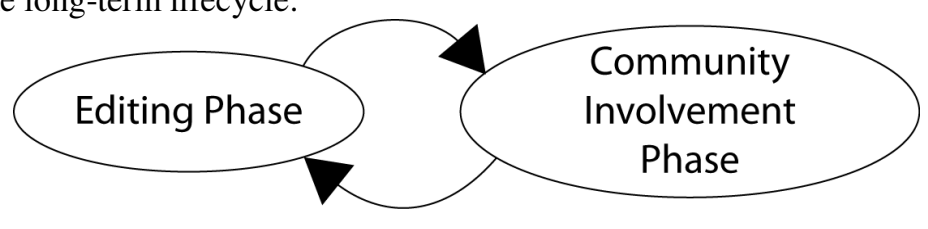

Fig. 7 - FEM Wiki Lifecycle

\section{Conclusion}

Web 2.0 tools have created a rich source of user-generated content on the Internet supporting the development of online communities of practise. The FEM wiki project is developing an online wiki version of the EPIET field epidemiology training programme, and an online community of practice providing a space for discussions for experts in field epidemiology. We undertook the task of defining the new editorial roles and content development processes in an online sphere to adapt the existing content for an online "wiki-like" use. This paper discusses the FEM wiki portal structure ensuring the ECDC-recognised content is available alongside user-generated and organically expanded pages. At the ehealth 2010 conference we will summarise the editorial process and discuss initial public user's activity after the opening of the site in November 2010. 
A long term aim of the project is to become the single-access portal for anyone with interests not only in field epidemiology nut for a wider communicable disease community around the world.

\section{Acknowledgements}

We acknowledge ECDC for funding this project, in particular, Arnold Bosman and Vladimir Prikazsky for managing the initiative; EPIET training programme for developing the initial chapters and FEM wiki Editorial Board, chaired by Julius Weinberg, and the Advisory Board, chaired by Mike Catchpole, for undertaking the editorial task. Further, we also acknowledge Lisa Lazareck, the coordinator of the Editorial Board, for all her efforts and enthusiastic editorial input; Christina Daly for coordinating administrative processes, Ed de Quincey for his work on the initial platform feasibility study for this project in 2009, and finally, CeRC researchers Dasun Weerasinghe and Gawesh Jawaheer for their involvement in the initial phase of the project.

\section{References}

1. Medicine 2.0: Social Networking, Collaboration, Participation, Apomediation and Openness. Eysenbach, G. 3, s.l. : Journal of Medical Internet Research, 2008, Vol. 20.

2. Cultivating Communities of Practice. Wenger, E., McDermott, R. and Snyder, W. s.l. : Harvard Business School Press, 2002.

3. Towards a collaborative learning environment for children's pain management: leveraging and online discussion forum. Curran-smith, J., Abidi, S. S. R. and Forgeron, P. 1, s.1. : Health Informatics Journal, 2005, Vol. 11.

4. Communities of Practice: Learning, Meaning and Identity. Wenger, E. s.l. : Cambridge University Press 2998.

5. Gannon-Leary, P.M. \& Fontainha, E. "Communities of Practice and virtual learning communities: benefits, barriers and success factors" ELearning Papers 26 Sept 2007 [Accessed Nov 2007]

6. User Information seeking behaviour: perceptions and reality. An evaluation of the WHO Lab resources Internet portal. Madle G., Berger A., Cognat S., Menna S., Kostkova, P.: Informatics for Health and Social Care, 2009 Jan;34(1):30-8

7. http://en.wikipedia.org/wiki/History_of_Wikipedia [Accessed June 2010]

8. SOMWeb: A Semantic Web-based System for Supporting Collaboration of Distributed Medical Communities of Practice. Falkman, G., Gustafsson, M., Jontell, M., Torgersson, O. (2008) Journal of Medical Internet Research 10(3):e25, Special Issue on Medicine 2.0. doi: 10.2196/jmir.1059.

9. http://www.facebook.com/press/info.php?statistics [Accessed June 2010]

10. Quincey, Ed de and Kostkova, Patty. Feasibility Study for the Field Epidemiology Manual (FEM wiki) pilot platform. I. s.l. : Internal Technical Report, 2009. 